\title{
Severe Vaginal Stenosis Simulating a Transverse Septum with Huge Hematocolpos in an HIV-patient: How to Make a Break in the Wall?
}

Attilio Di Spiezio Sardo*, Marialuigia Spinelli, Divina D’Auria, Brunella Zizolfi, Matilde Sansone, Carmine Nappi and Costantino Di Carlo

Department of Gynaecology and Obstetrics, University of Naples "Federico II , Via Pansini 5, 80131 Naples, Italy

\begin{abstract}
Introduction: Vaginal infections are the most commonly reported condition among women with HIV/AIDS and such infections often represent the most common initial manifestation of the illness. As a consequence, post-infective early and late complications are frequently reported in these patients. Vaginal stenosis has been reported as a rare late complication of severe vaginal infections. The vaginoscopic treatment with miniaturized instruments of a severe vaginal stenosis with huge hematocolpos in a HIV-infected woman is here reported.

Case presentation: A 38 year-old HIV-infected woman was referred to our Emergency Unit because of severe pelvic pain, increasing over time, beginning a week before, after a sexual intercourse. At physical and ultrasonographic examination, complete vaginal stenosis up to the vaginal vestibule (miming a transversal vaginal septum) with a giant haematocolpos was diagnosed. She was scheduled for traditional surgical treatment in the operating room with Hegar dilatators but the procedure was unsuccessful. The patient was then scheduled for an operative vaginoscopy in office setting by means of a $5 \mathrm{~mm}$ oval-profile hysteroscope. The use of $5 \mathrm{Fr}$ bipolar electrode and mechanical instruments allowed to make a break in the "wall", thus allowing the complete drainage of the haematocolpos without any significant complication.
\end{abstract}

Conclusion: Vaginoscopy with miniaturized instruments represent a safe, convenient, and efficient therapeutic modality that can be used in patients with a restrictive vaginal opening or narrow vaginal canal.

Keywords: HIV; Vaginal; Stenosis; Vaginoscopy; Hematocolpos; Hysteroscopy; Infection

\section{Introduction}

Vaginal infections, as well as being the pathological condition more frequently described in women with HIV/AIDS, are very often the initial manifestation of the disease [1]. Many types of vaginal infections are twice as common in women who have HIV than in women who are HIV-negative. Furthermore, immuno-suppression due to HIV infection can lead to genital infections that are unusually more severe and difficult to treat [2]. However, the exact mechanisms by which the presence of the HIV virus can affect lower female genital tract remain still debated in the scientific community [3].

The most common vaginal infections, which have all been reported to occur more frequently among HIV-infected women, are either fungal infection or candidal infection and bacterial and viral infection such as chlamydia, gonorrhea and genital herpes. The symptoms associated with these infections are particularly severe in HIV-infected women and include leucorrhea, redness, soreness, bleeding and/or dyspareunia.

As a consequence, post-infective both early and late complications are more frequent in these patients, such as post-abortion pelvic inflammatory disease and cuff cellulitis in patients undergoing gynecologic surgery. Vaginal stenosis has been reported as a rare late complication of severe vaginal infections.

We here report the treatment by operative vagino-hysteroscopy with miniaturized instruments of a complete vaginal stenosis up to the vaginal vestibule, with a giant haematocolpos in a immuno-deficient HIV positive woman.

\section{Case Presentation}

A 38 year-old HIV-infected woman, was referred to the Emergency Room of our Department of Obstetrics and Gynecology for severe pelvic pain, increasing over time, started a week before, after a sexual intercourse (the first after a 6 year period of sexual abstinence). The patient referred that in the last two months started to complain of episodically cramping pain in the low-pelvis, but that, during and immediately after the sexual intercourse, it became ingravescent and intractable, propagating itself to the vaginal and low-back areas too.

The patient had a long-term drug abuse anamnesis, with severe addiction for opioid and cocaine. Her past medical history included lung abscess, HCV-related chronic liver disease, recurrent ( $>4$ episodes/year) yeast infections and severe pelvic inflammatory disease. The yeast infections were mucocutaneous candidiasis and occurred as oropharyngeal and vulvovaginal diseases. These latter were particularly frequent in the last 5 years ( $>7$ episodes/year) and presented with marked itching, vaginal erythema with adherent white discharge and swelling of labia and vulva with pustulopapular peripheral lesions. Topical preparations of nystatin and fluconazole tablets were administrated to treat these infections, while a chronic assumption of oral lactic ferments was started in the last three years to prevent them.

She had undergone three cesarean sections and surgical treatment of sacro-coccygeal fistula in childhood. She was in amenorrhea for the last 4 years, but she had never manifested climacteric symptoms.

At gynecological examination the vaginal vestibule was completely obstructed due to the presence of a sort of inaccessible wall (similar to a complete fibrotic transversal vaginal septum).

*Corresponding author: Attilio Di Spiezio Sardo, Department of Gynaecology and Obstetrics, University of Naples "Federico II , Via Pansini 5, 80131 Naples, Italy, Tel: 3939889647; E-mail: cdispie@tin.it

Received February 03, 2014; Accepted March 24, 2014; Published March 26 2014

Citation: Sardo ADS, Spinelli M, D'Auria D, Zizolfi B, Sansone M, et al. (2014) Severe Vaginal Stenosis Simulating a Transverse Septum with Huge Hematocolpos in an HIV-patient: How to Make a Break in the Wall? J Clin Case Rep S1: 003. doi:10.4172/2165-7920.S1-003

Copyright: (c 2014 Sardo ADS, et al. This is an open-access article distributed under the terms of the Creative Commons Attribution License, which permits unrestricted use, distribution, and reproduction in any medium, provided the original author and source are credited. 
Vaginal inspection was not possible and speculum could not be inserted into her vagina due to the significant foreshortening occlusion. Vaginal exploration was impossible even with a pediatric swab.

Trans-abdominal ultrasonography showed an oval area of $101 \times$ $88 \times 78 \mathrm{~mm}$ in the pelvic region, centrally below the cervix, suggesting a huge hematocolpos (Figure 1).

After further investigations, the patient clarified that the sexual intercourse she had 7 days before was not a vaginal one, but rather an anal intercourse, as confirmed also by her partner.

She was scheduled for traditional surgical treatment in the operating room with Hegar dilatators but the procedure was unsuccessful because of the hard consistency of the tissue obstructing the vaginal opening and the fear of the surgeon to create damage with blind maneuvers to the bladder and/or rectum as consequence of a totally distorted anatomy in vulvo-vaginal area.

Therefore, the patient was scheduled for an alternative surgical approach in office setting with hysteroscopic instrumentation and vaginoscopic technique [4]. Vagino-hysteroscopy was performed using a miniaturized $5 \mathrm{~mm}$ continuous flow office hysteroscope (Bettocchi Office Hysteroscope size 5; Karl Storz, Germany) with a $30^{\circ}$ grade optic and an incorporated $5 \mathrm{Fr}$ working channel. Normal saline solution was used for vaginal and uterine distension. Vaginal pressure was maintained at a constant 30 to $40 \mathrm{~mm} \mathrm{Hg}$ with an electronic pump for irrigation and aspiration (Endomat; Karl Storz GmbH \& Co, Tuttlingen, Germany). A tablet of a FANS was administered to the patient 1 hour before the procedure. Vulvar labia were maintained close during the procedure in order to obtain an adequate distension of the vaginal vestibule.

First, a 5Fr grasping forceps was used to test the assignability of the tissue. Then, a 5Fr bipolar electrode (Versapoint Twizzle electrode, Gynaecare) has been used in a cold way (i.e. without electricity) as a sort of needle just to create a little hole in the central area, maintaining an adequate safe distance with the surrounding organs (the bladder above and the rectum below); as soon as a "black hole" was created and blood started to come out (Figure 2A), the electrode was then activated in order to perform four radial incisions (1-2 $\mathrm{mm}$ deep), at approximately 3, 6, 9 and 12 o'clock positions of the "black hole" (Figure 2B). Then, $5 \mathrm{Fr}$

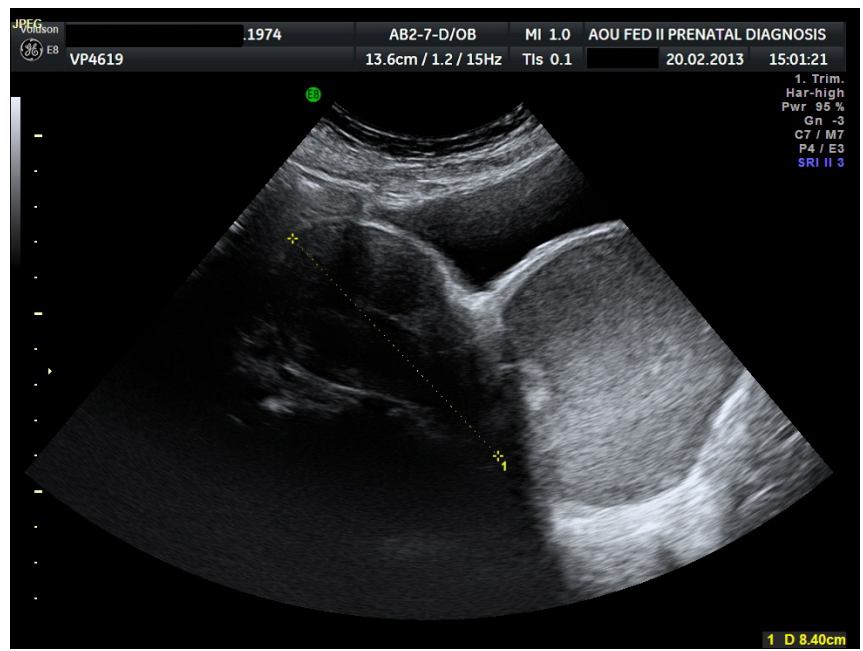

Figure 1: Trans-abdominal ultrasonography showed an oval area of $101 \times 88$ $\times 78 \mathrm{~mm}$ in the pelvic region, centrally below the cervix, suggesting a huge hematocolpos.

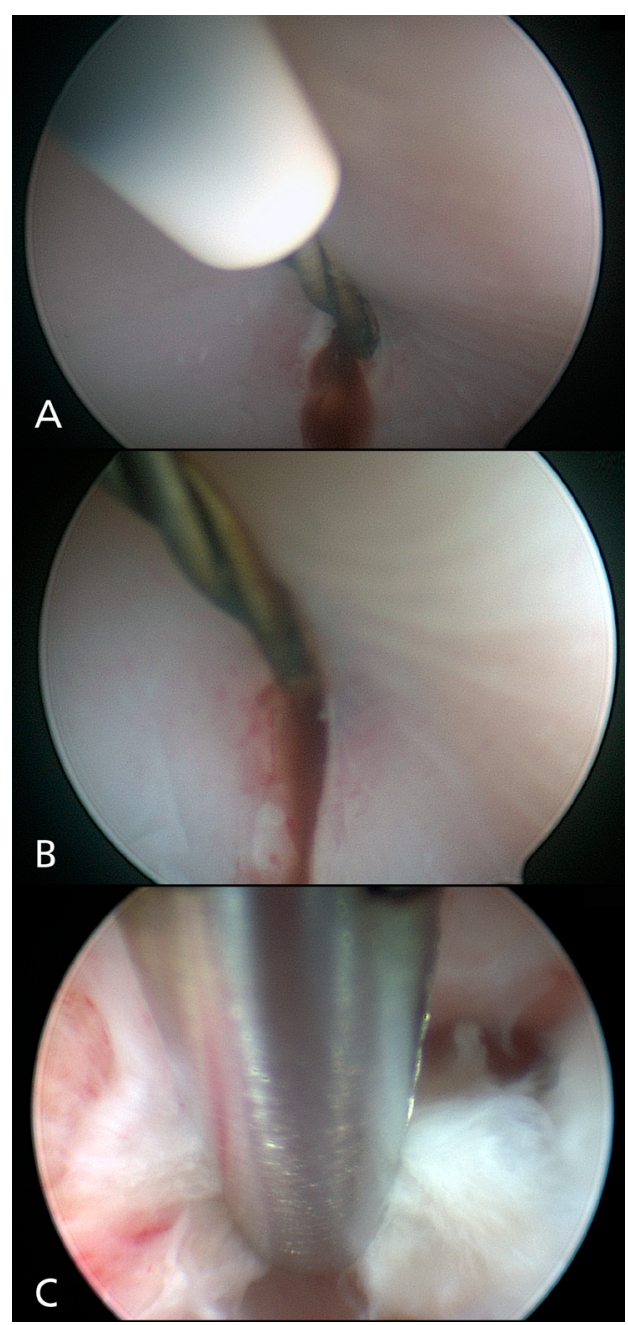

Figure 2: A. As soon as a "black hole" was created and blood started to come out. B: The electrode was then activated in order to perform four radial incisions (1-2mm deep), at approximately 3, 6, 9 and 12 o'clock positions of the "black hole". C: Then 5Fr grasping forceps with teeth were inserted within the opening.

grasping forceps with teeth were inserted within the opening with the jaws first closed (Figure 2C), and then gently opened in order to stretch the fibrotic tissue up to 3-4 mm. A complete drainage of hematocolpos was then achieved after cervical dilatation up to Hegar 10 was carried out. Finally, the complete vaginal patency was maintained by inserting a Foley catheter (left inside for 3 days) (Figure 3).

The postoperative period was completely uneventful, and complete drainage of hematocolpos was confirmed by ultrasonography performed the day after surgery and one week later.

The patient was then followed for 6 months through monthly telephone consultations, gynecological examinations and ultrasonographies performed every three months, which confirmed a normal patent vagina, a satisfactory sex life (that started about 20 days after surgery) and no recurrence of the hematocolpos.

\section{Discussion}

Vaginal stenosis have been defined as the inability to pass two fingers into the vagina [5], however, according to a broader definition, they represent the narrowing and/or loss of flexibility of the vagina, 


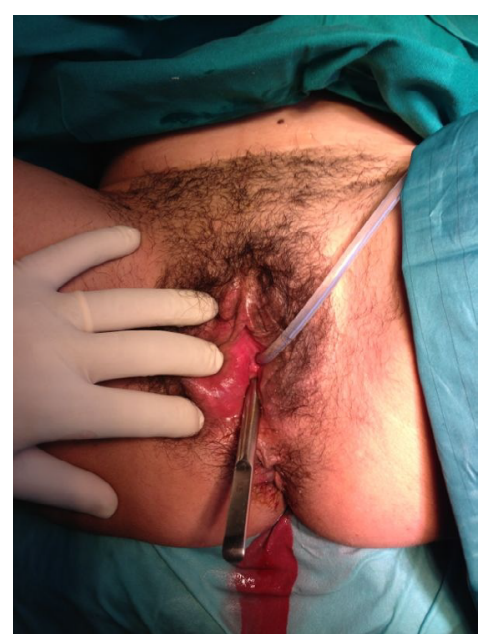

Figure 3: The complete vaginal patency was maintained by inserting a Foley catheter (left inside for 3 days).

often accompanied by other changes such as the dryness and loss of resilience of scar tissue.

Vaginal stenosis can lead to long-term effects on sexual activity, including vaginal dryness, pain, and dyspareunia, thus impairing, ultimately, the quality of life. Women who experience painful intercourse will probably be less likely to pursue vaginal sexual activity, and less use of the vagina can lead to further shortening and narrowing, though there is little formal literature adequately covering this problem $[6,7]$.

Frequently, an adequate clinical evaluation may be challenging, because vaginal examination may result very painful in such cases $[8,9]$.

Most of vaginal shortening are reported as a late consequence of severe vaginal infections, and as being greater for women with cervical cancer, endometrial cancer, and in those undergoing radiotherapy [8-10].

The main interest of our case is in that, to our knowledge, vaginal stenosis in patients with HIV has never been previously reported in the worldwide literature; this may be surprising, since immunosuppression due to HIV infection can lead to genital infections that are unusually more severe and difficult to treat.

Vaginal stenoses are generally difficult to manage and tend to recur despite proper treatment. Currently, there are several retrospective reviews of surgical intervention for vaginal stenosis or shortening. Most reported the use of split-thickness skin grafts to lengthen a foreshortened vagina (especially after treatment for gynecologic cancers) [11-13]. Other Authors evaluated the use of myocutaneous grafts to reconstruct the perineum and/or posterior vagina or the use of dilatators $[14,15]$.

Our case is peculiar in that an alternative approach has been used for the management of such a condition, since standard surgical approach with dilatators was not feasible.

Vaginoscopy refers to use of an instrument, other than a speculum, to visualize the vaginal canal. It has been described in the literature as far back as the 1950s and continues to be used for diagnosing and treating vaginal pathologies/conditions. It has also been used for evaluating pelvic trauma and infection [15].

To the best of our knowledge, this case reports the first successful office treatment of a severe vaginal stenosis in a HIV-affected woman by means of operative vaginoscopy.

The main advantage of an office approach without any kind of anesthesia lies in the prompt identification of false passages or damage to surrounding organs. Indeed, a sudden increase of patient's pain represents a warning sign that a false passage into the vagina or damage to other innervated structures (i.e. bladder, rectum) has been creating. Indeed no sensitive nerve terminals have been demonstrated in the fibrous tissue.

Currently, a wide set of 5Fr mechanical and electrified instruments may be used to overcome vaginal and cervical stenosis. In particular, we used a bipolar electrode for accessing the stenotic vaginal cavity, since the severity of the stenosis precluded the use of bigger mechanical instruments. A needle-like bipolar electrode was used first to create a point of access in a "cold way" and then to cut the fibrotic tissue around the neo-formed "hole" in a safe way by performing several radial incisions. The mildest energy of vapour cutting mode was used (VC3 $50 \mathrm{~W}$ ), since it provided the lowest energy flow into the tissue, thus reducing tissue traumatism. In this way, a breech was created in the vaginal wall and cervical dilatators could be used to enlarge this opening and to successfully drain the enlarged vaginal cavity.

The efficacy of such innovative approach of vaginal stenosis was confirmed by post-operative diagnostic hysteroscopy and 3D-TVS, that showed that the procedure produced good anatomical results with the complete drainage of hematocolpos while restoring vaginal patency. The rapid return to normal activities and sexual life of the woman completed the success of the procedure.

\section{Conclusions}

Vaginoscopy with miniaturized instruments represent a safe, convenient, and efficient therapeutic modality that can be used in patients with a restrictive vaginal opening or narrow vaginal canal because offers minimal traumatism and risk of recurrence.

\section{References}

1. Cu-Uvin S, Hogan JW, Warren D, Klein RS, Peipert J, et al. (1999) Prevalence of lower genital tract infections among human immunodeficiency virus (HIV)seropositive and high-risk HIV-seronegative women. HIV Epidemiology Research Study Group. Clin Infect Dis 29: 1145-1150.

2. Watts DH, Springer G, Minkoff H, Hillier SL, Jacobson L, et al. (2006) The occurrence of vaginal infections among HIV-infected and high-risk HIVuninfected women: longitudinal findings of the women's interagency HIV study. J Acquir Immune Defic Syndr 43: 161-168.

3. Shacklett BL, Greenblatt RM (2011) Immune responses to HIV in the female reproductive tract, immunologic parallels with the gastrointestinal tract, and research implications. Am J Reprodlmmunol 65: 230-241.

4. Bettocchi S, Selvaggi L (1997) Avaginoscopic approach to reduce the pain of office hysteroscopy. J Am Assoc Gynecol Laparosc 4: 255-258.

5. Nunns D, Williamson K, Swaney L, Davy M (2000) The morbidity of surgery and adjuvant radiotherapy in the management of endometrial carcinoma. Int $\mathrm{J}$ Gynecol Cancer 10: 233-238.

6. Brand AH, Bull CA, Cakir B (2006) Vaginal stenosis in patients treated with radiotherapy for carcinoma of the cervix. Int J Gynecol Cancer 16: 288-293.

7. Judith K. Wolf, MD (2006) Prevention and treatment of vaginal stenosis resulting from pelvic radiation therapy. The University of Texas M. D. Anderson Cancer Center, Houston, TX.Commun Oncol 3: 665-671

8. Flay LD, Matthews JH (1995) The effects of radiotherapy and surgery on the sexual function of women treated for cervical cancer. Int $\mathrm{J}$ Radiat Oncol Biol Phys 31: 399-404.

9. Hartman P, Diddle AW (1972) Vaginal stenosis following irradiation therapy for carcinoma of the cervix uteri. Cancer 30: 426-429. 
Citation: Sardo ADS, Spinelli M, D’Auria D, Zizolfi B, Sansone M, et al. (2014) Severe Vaginal Stenosis Simulating a Transverse Septum with Huge Hematocolpos in an HIV-patient: How to Make a Break in the Wall? J Clin Case Rep S1: 003. doi:10.4172/2165-7920.S1-003

10. MacLeod C, Fowler A, Duval P, D'Costa I, Dalrymple C, et al. (1999) Adjuvant high-dose rate brachytherapy with or without external beam radiotherapy posthysterectomy for endometrial cancer. Int J Gynecol Cancer 9: 247-255.

11. Morley GW, DeLancey JO (1991) Full-thickness skin graft vaginoplasty for treatment of the stenotic or foreshortened vagina. Obstet Gynecol 77: 485-489.

12. Hyde SE, Hacker NF (1999) Vaginal reconstruction in the fibrotic pelvis. Aust N Z J Obste Gynaecol 39: 448-453.

13. Roberts WS, Hoffman MS, LaPolla JP, Ruas E, Fiorica JV, et al. (1991)
Management of radionecrosis of the vulva and distal vagina. Am J Obstet Gynecol 164: 1235-1238.

14. Germann G, Cedidi C, Petracic A, Kal ᄀlinowski F, Herrfarth C (1998) The partial gluteus maximusmusculocutaneous turnover flap. An alternative concept for simultaneous recon-struction of combined defects of the posterior perineum/ sacrum and the posterior vaginal wall. Br J Plast Surg 51: 620-623.

15. Skene Al, Gault DT, Woodhouse CR, Breach NM, Thomas JM (1990) Perineal, vulval and vaginoperineal reconstruction using the rectus abdominismyocutaneous flap. Br J Surg 77: 635-637.
This article was originally published in a special issue, Obstetrics and Gynecology handled by Editor. Dr. Yigit Cakiroglu, Kocaeli University, Turkey 